\title{
Assessing the biomass of shrubs typical of Mediterranean pre-forest communities
}

\author{
P. CORONA ${ }^{1}$, S. PASTA ${ }^{2}$, G. GIARDINA ${ }^{2}, \&$ T. LA MANTIA ${ }^{2}$ \\ ${ }^{1}$ DIBAF, University of Tuscia, via San Camillo de Lellis snc, 01100 Viterbo, Italy and ${ }^{2}$ DEMETRA, \\ University of Palermo, viale delle Scienze, Ed. 4, 90128 Palermo, Italy
}

\begin{abstract}
One of the most outstanding issues of current environmental research is the need of reliable assessments of carbon stock (i.e. above-ground (a.g.) and below-ground biomass, deadwood, litter, and organic soil matter) within forest ecosystems. Although shrub vegetation plays an important role in accumulating carbon in many Mediterranean environments, there is still very little knowledge on the carbon they store. In this article, we analyze the a.g. carbon stock of several Mediterranean shrubby communities in Sicily (Italy), dominated by Pistacia lentiscus, Chamaerops humilis, Euphorbia dendroides and Spartium junceum. Plant samples for each species were selected, and morphometric attributes (height, crown diameter, diameter at breast height, etc.) were measured to establish models for estimating a.g. biomass. Dendrometric data were also used to support the assessment of shrub biomass at community level by coupling them with the coverage of the considered woody species estimated from several hundreds of phytosociological relevés carried out on pre-forest Sicilian communities. Although the results of such an approach have to be considered as rough assessments, the trial proves interesting, given the lack of information on stand biomass of pre-forest vegetation within the Mediterraean region.
\end{abstract}

Keywords: Pistacia lentiscus, Chamaerops humilis, Euphorbia dendroides, Spartium junceum, shrub inventory

\section{Introduction}

The assessment of above-ground (a.g.) biomass within Mediterranean forest ecosystems is relevant for both scientific and practical purposes. In fact, it is necessary to support the study of the global carbon cycle in this region, as it can provide useful information for planning and managing these ecosystems (Corona \& Marchetti 2007). With respect to the decisions taken to face climate change, the estimation of the carbon stored by pre-forest formations, which are quite widespread under Mediterranean environments, is of major interest in accordance with international agreements on the enforcement of the Kyoto Protocol. Although the increase of these plant communities is often due to dynamic processes that occur after land abandonment (Corona et al. 2008; La Mantia et al. 2008) as a result of environmental and land use policies, biomass derived from revegetation ought to be included in the calculation of the total carbon amount. According to the "Marrakesh Agreements": "... revegetation is a human-induced activity to increase local carbon stocks through the establishment of vegetation that covers a minimum area of 0.05 hectares and does not meet the definitions of afforestation and reforestation...". However, it is usually difficult to obtain reliable data on biomass of pre-forest formations. This is due to, inter alia: (i) the lack of experimental data on the shrub species and (ii) the highly variable composition of shrub communities. Another problem, at least for many Mediterranean countries, is related to the lack of information on the coverage of pre-forest (shrub) formations.

An overview of the studies on shrub biomass in Mediterranean environments has been recently carried out by Costa \& La Mantia (2005). In particular, these studies concentrated on several typical Mediterranean plant communities such as high (with Quercus ilex, Erica arborea, Phillyrea 